\title{
Estrategia educativa para la formación de recursos humanos en la gestión de información en salud
}

\section{Educational strategy for the formation of human resources in health information management}

\author{
María Vidal Ledo'; Alfredo Rodríguez Díaz ";Ariel Delgado Ramos"; \\ Eduardo Manrique García'"' \\ 'Máster en Informática en Salud. Escuela Nacional de Salud Pública. La Habana, \\ Cuba. \\ "Profesor Auxiliar. Escuela Nacional de Salud Pública. La Habana, Cuba. \\ "'IMáster en Microelectrónica. Asistente. Centro de Enseñanza de Cibernética \\ Aplicado a la Medicina (CECAM) La Habana, Cuba.
}

\section{RESUMEN}

La necesidad de formar recursos humanos en la gestión de información en salud implica disponer de una estrategia educativa útil para estos fines. La estrategia que se desarrolla en Cuba responde a las características de la Universidad Cubana: científica, tecnológica y humanista, con un fuerte compromiso social. Basado en este modelo se presenta una estrategia educativa que tiene en cuenta las necesidades de las competencias y el desempeño que se requieren en el personal de la salud para el tratamiento de la información. Se muestra sus ejes de formación, arquitectura y características del diseño. La estrategia propuesta prevé los procesos capacitantes o de habilitación en los servicios, la formación básica o de pregrado y define diferentes figuras educativas para el perfeccionamiento, la especialización y maestría de estos profesionales; es integral y pertinente y contribuye a obtener un graduado de perfil amplio, competente en las disciplinas de informática, estadísticas sanitarias, información científica y otras. Esta estrategia educativa se aplica en el marco del desarrollo del sector de la salud y de la sociedad cubana con amplio apoyo y voluntad política y finalmente garantiza el recurso humano requerido en cada puesto de trabajo, así como sistemas informáticos integrados para una adecuada gestión de la información en el sector. 
Palabras clave: Docencia, pregrado, formación de recursos humanos, capital humano, gestión de información y conocimiento.

\begin{abstract}
The need for the formation of human resources in health information management implies the availability of a useful educational strategy to these ends. The strategy followed by Cuba responds to the characteristics of the Cuban university, that is, scientific, technological, humanistic and socially compromised. On the basis of this model, an educational strategy was submitted, which takes into account the competencies and performance that the health care staff requires for information processing. The axes of formation, architecture and design characteristics were presented. The suggested strategy foresees the enabling processes at service, the basic or undergraduate formation, defines the various educational figures for upgrading, specialization and master's degree of these professionals; it is comprehensive and relevant and contributes to the formation of a broad profile graduate who is very competent in informatics, health statistics, scientific information and other branches. This educational strategy is implemented as part of the development of the health care sector and of the Cuban society, with wide political support, and finally it assures the required human resources for each work post as well as the integral informatics systems to attain suitable health information management.
\end{abstract}

Key words: Teaching, undergraduate, human resource formation, human capital, information and knowledge management.

\title{
I NTRODUCCI ÓN
}

El Sistema Nacional de Salud (SNS), por su misión, de acuerdo al enfoque estratégico y programático que mantiene, requiere de un constante flujo informativo que permita mantener un alto nivel de conocimiento de cada una de las actividades que se realizan desde la base hasta el nivel central. Su organización y conducción, requiere de personal entrenado que intervenga en los procesos de dirección y toma de decisiones en la asistencia e investigación en función de la gestión de información que se solicita para garantizar la satisfacción de estas necesidades. ${ }^{1}$

La formación de recursos humanos propios se realiza sistemáticamente en el SNS y ha formado parte intrínseca de su propia evolución. Para ello cuenta de una Red de establecimientos destinados a la formación de recursos humanos para las ciencias médicas, estomatológicas y de tecnología de la salud. En esta red de instituciones se han formado compañeros en más de 18 especialidades tecnológicas, entre ellas los Técnicos en Bibliotecología Médica y Técnicos en Estadísticas de Salud y Registros Médicos. ${ }^{1}$ 
La formación de competencias en estas áreas de desempeño se ha realizado en Cuba desde la década del 60 del pasado siglo hasta el año 1991 e incluía el desarrollo de habilidades en las herramientas informáticas que existían para la época. A partir de la década del 90 se tomó la decisión de cerrar estas carreras y utilizar los técnicos comunes en bibliotecas que se formaban en las escuelas de cultura y educación, y para las estadísticas sanitarias los que procedían de los tecnológicos de economía del Ministerio de Educación (MINED) ${ }^{2}$ Estos graduados no satisficieron las necesidades, ya que no existía una orientación vocacional clara y su preparación general no se ajustaba a las necesidades del SNS. ${ }^{3}$

La sociedad cubana actual enfrenta la convergencia tecnológica en la informática, la microelectrónica y las telecomunicaciones, a través de un proceso de informatización social que pretende socializar estas tecnologías, en cuyo reto están comprometidos todos los sectores socioeconómicos y por ende la Salud Pública Cubana, en que se aprecia una nueva visión cualitativa de la información donde ella actúa de forma catalizadora y modificadora en las estructuras del conocimiento, en el proceso de comunicación social en que están presentes las influencias del entorno donde se crea, recupera y utiliza; lo cual define un contexto propiciador para cambios en métodos y estilos de trabajo. ${ }^{4}$

Unido a ello el desarrollo de los Sistemas de Gestión de la Información y el Conocimiento para la Dirección en Salud en su sentido amplio, requiere de herramientas modernas de dirección, caracterizadas por los Sistemas de Información de Salud (SIS) ${ }^{5}$ y las Tecnologías de la Información y las Comunicaciones (TIC), y por ende, de un profesional capaz de gerenciarlas, entendiendo que lo que se gerencia no es el conocimiento, sino las condiciones en que este es compartido, y disponga de los beneficios de accesibilidad, disponibilidad y rapidez, de manera que los directivos y profesionales en general puedan lograr la mayor eficiencia y eficacia en su gestión.

\section{PREMI SAS}

La estrategia de informatización del sector de la salud en Cuba reclama, por ende, un personal preparado en las TIC y en la gestión de información y conocimiento, que participe de manera activa en el proceso de cambio que el SNS demanda acorde a la sociedad de la información contemporánea y que cuenta con apoyo y voluntad política para lograrlo. Es por ello que se define como la primera de las cinco tareas principales de esta estrategia el "Proyecto Horizontal de Formación, Preparación y Perfeccionamiento de Recursos Humanos", ${ }^{6-8}$ con un enfoque participativo, teniendo en cuenta, como ideas rectoras, tres ejes de formación: ${ }^{7}$

- Formación en valores.

- Vinculación del estudio con el trabajo.

- Sólida formación teórico-práctica.

Se partió de la identificación de necesidades de aprendizaje sobre las TIC y los SIS según cargo, evaluando los diferentes puestos de trabajo en las entidades, servicios y departamentos de registros médicos y estadísticas sanitarias, vigilancia en salud, economía, información científico-técnica e informática, que permitiera definir un modelo pedagógico ${ }^{7}$ basado en: 
- Perfil amplio de formación, que cubriera los requerimientos del SNS.

- Formación capacitante, profunda formación básica y continuidad especializada.

- Independencia, creatividad y resolutividad ante los problemas de los servicios.

- Plan de estudios estructurado y contextualizado a la realidad de cada lugar.

- Procesos educativos centrado en el estudiante, con un aprendizaje autónomo, integral y significativo, basado en problemas, donde el estudiante sea objeto y sujeto de su propio aprendizaje, comprenda y construya su propio conocimiento.

- Nuevo rol del profesor quien debe orientar esa actividad con el fin de lograr que el alumno, desarrolle su pensamiento crítico-reflexivo e integre los contenidos objeto de su aprendizaje, en el marco de los valores, la cultura y contexto social en que se va a desempeñar.

- Momentos de certificación para cada proceso capacitante, ciclos de formación básica y modalidades de educación permanente, que permita la continuidad de estudios independientemente del lugar o nivel del SNS donde se encuentre el estudiante.

Este desarrollo no puede verse fuera del contexto de las tendencias de cambio y desarrollo general de la educación superior en Cuba, en América Latina y el Mundo $^{9,10}$ y la repercusión que ellas tienen en el proceso que se manifiesta en la salud.

En este entorno se inserta el diseño de esta estrategia educativa que debe responder a los retos y exigencias de transformación y cambio que se promueven especialmente en el sector de la salud y en la sociedad en su conjunto.

\section{ESTRATEGI A EDUCATI VA}

La estrategia educativa que se desarrolla en Cuba responde a las características de la Universidad Cubana: científica, tecnológica y humanista, con un fuerte compromiso social, que garantiza la oportunidad a todas las personas del país de acceder a la educación media y superior sin límites ni restricciones, cuyo paradigma está en:

Brindar a la sociedad un profesional formado de manera íntegra, profesionalmente competente, con preparación científica para aceptar los retos de la sociedad moderna y con un amplio desarrollo humanístico para vivir en la sociedad de esta época y servirla con sencillez y modestia, con los valores como pilar fundamental de su formación. ${ }^{10}$

Basado en el modelo cubano, de acuerdo a las necesidades de las competencias y el desempeño que se requieren en el personal de la salud para el manejo de la información, dirigida fundamentalmente al conocimiento del estado de salud de la población y la toma de decisiones para la conducción de los procesos y actividades del SNS, se diseñó la estrategia cuya arquitectura fue enfocada como un sistema que presenta tres subsistemas integrados por una disciplina rectora: la gestión de información en salud. ${ }^{7,11}$ Estos subsistemas lo constituyen los: 
- Procesos capacitantes.

- Formación básica.

- Formación permanente.

Los procesos capacitantes están dirigidos a trabajadores con un nivel de escolaridad de 9 no. y 12 mo. grados, con el objetivo de habilitarlos y prepararlos en los conocimientos generales requeridos para su desempeño como auxiliares en departamentos y servicios de estadísticas y registros médicos, información científica y bibliotecas, e informática básica en salud. Se imparte desde el puesto de trabajo, en la propia institución donde labora el trabajador.

La formación básica o de pregrado, se realiza a partir de educandos procedentes de diversas fuentes del sistema regular de enseñanza, técnicos y trabajadores de los servicios de salud afines, con nivel de $12 \mathrm{mo}$. grados. Tiene un currículo flexible y se dirige a la formación en conocimientos básicos en las disciplinas de humanidades, registros médicos y estadísticas de salud, información científico-técnica y bibliotecología y tecnologías e informática, afines a los servicios para los que se forman y en los cuales quedan insertados como trabajadores, de no serlo, a partir del 2 do. año, con un modelo de perfil amplio que en su desarrollo permite alcanzar los títulos de:

- Técnico básico en Gestión de Información en Salud (un año de formación).

- Técnico medio en Gestión de Información en Salud (dos años de formación).

- Licenciado en Tecnología de la Salud en el perfil en Gestión de Información en Salud (dos años de formación).

Se imparte por encuentros en sedes universitarias territoriales del sistema de ciencias médicas, en la modalidad semipresencial, con el uso de entornos virtuales de enseñanza-aprendizaje y trabajo en red. Existe también una edición virtual en la universidad virtual de salud (UVS) para aquellos estudiantes que cumplen funciones en el exterior del país como parte del Programa Integral de Salud Cubano.

La formación permanente o continuada, brinda a este profesional de perfil amplio las opciones de continuidad de estudio para su perfeccionamiento a través de cursos cortos y diplomados virtuales, la especialización en dirección en los servicios de salud, bioestadística, seguridad informática, gestión de información y el conocimiento, así como, alcanzar grados científicos a partir de las maestrías en informática en salud, gestión de información en salud y otras salidas que se estudian para ellos.

La arquitectura de esta estrategia educativa pone de manifiesto la filosofía y el contexto en que se desarrolla de acuerdo a las ideas rectoras, la misión de la educación superior y la salud pública cubana, ${ }^{12}$ su enfoque por competencias se basa en el análisis de las funciones que deben ser desplegadas en cada puesto de trabajo de los servicios de salud; el modelo pedagógico definido según las necesidades de aprendizaje, modalidades y aplicación de las nuevas tendencias de la educación moderna; el rol de los profesores, así como el plan de estudios en cuanto al desarrollo de contenidos, métodos, recursos de aprendizaje, evaluación y certificación; con un enfoque curricular sistemático, flexible, pertinente, transversal, contextualizado, integrado e investigativo, definidos en sus objetivos educativos e instructivos, que aborda el problema de los servicios de salud según 
modalidad presencial o virtual con cuatro disciplinas: humanidades, registros médicos y estadística de salud, información científica y bibliotecología y tecnologías e informática, el cual es sometido a una evaluación interna y externa en todos sus procesos. $^{7}$

Un aspecto relevante del modelo pedagógico son los procesos de enseñanzaaprendizaje de forma interdisciplinaria, con la aplicación de las tendencias modernas de la construcción del conocimiento, ${ }^{13}$ centrado en el estudiante, cuyo perfil formativo es aprender a aprender, aprender-haciendo y aprender a ser el profesional a que se aspira, basado en métodos crítico-reflexivos que mantienen como principio central la investigación-acción e incluye nuevos conceptos de desarrollo profesional donde los sujetos de aprendizaje no pueden estar separados de su acción y de su vivencia, asentándose en el principio de reflexionar sobre la propia práctica como una instancia ineludible de innovación y transformación de los procesos educativos para autorregular la intervención y el rol docente según las condiciones objetivas y subjetivas del entorno en que se aplica.

Otro elemento importante en la estrategia es el uso intensivo de las TIC y su aplicación al trabajo en red en todos los procesos de gestión, procesamiento, publicación y conservación de los SIS, así como su integración al proceso docente educativo, de manera que el alumno hace real uso de la tecnología en cualquiera de los subsistemas en que se encuentre, para el desarrollo de habilidades que le permita explotarlas en su desempeño profesional a fin de lograr la excelencia en su trabajo y de esta manera repercuta en los resultados de los servicios de salud.

Esta estrategia integrada al desarrollo del SNS, que ya cuenta con sus primeros graduados en ocho provincias del país, permite formar líderes y especialistas en el campo de la información y la informática de salud y cubrir las necesidades de diferentes campos y perfiles ocupacionales dentro del sector:

- Registros médicos y estadísticas de salud.

- Bibliotecología médica e información científico-técnica.

- Informática en salud.

- Seguridad informática.

- Dirección en los procesos de gestión de información en los sistemas y servicios de salud.

- Docencia e investigación.

Existe también la formación regular de recursos humanos en ciencias informáticas, formados en el sistema regular de enseñanza por la Universidad de Ciencias Informáticas, ${ }^{8}$ donde está la Facultad para la Salud, que desarrolla sistemas informáticos de alta complejidad para las instituciones de salud cubanas, concebidos dentro del desarrollo estratégico del SNS sin contradicciones antagónicas, ya que el personal propio adquiere gran cultura tecnológica y conoce profundamente los procesos del sector y esto le permite ser un interlocutor apropiado a los desarrolladores sistemas informáticos de alto nivel aportando un intercambio y sinergia en función de lograr mejores resultados desde su óptica de usuario experimentado. 
Se puede concluir que este modelo integra un proceso que contempla la habilitación, preparación, formación y perfeccionamiento de recursos humanos en gestión de información en salud, con la aplicación de técnicas y métodos de enseñanza modernos, con el uso intensivo de las tecnologías y el trabajo en red como forma de integrar contenidos, desarrollar habilidades e incentivar una estrategia progresiva del proceso de enseñanza-aprendizaje en las competencias requeridas para lograr mejores desempeños, donde la práctica social y valores éticos se pongan de manifiesto y constituyan el eje principal educativo que logre cubrir las necesidades de aprendizaje en esta esfera para el SNS.

Contribuye a obtener un graduado de perfil amplio, especializado en las disciplinas de informática, estadísticas sanitarias e información científica en función de un mejor desempeño en las actividades de gestión de información en salud e integrado a las estrategias de desarrollo del SNS. Además, contribuye a la formación y capacitación posgraduada en la búsqueda de una especialización y una mejor preparación.

Esta estrategia educativa se aplica en el marco del desarrollo estratégico del sector de la salud y la sociedad cubana con amplio apoyo y voluntad política para lograr sus objetivos, permite lograr técnicos y profesionales que interactúen con otros en los servicios de salud y otros escenarios técnicos y que den soluciones a las necesidades prácticas, presentes y futuras del SNS. Garantiza el recurso humano requerido en cada puesto de trabajo, así como, sistemas informáticos integrados para una adecuada gestión de la información en el desarrollo del sector.

\section{REFERENCI AS BI BLI OGRÁFI CAS}

1. Vidal Ledo M. Evaluación del diseño curricular del perfil de Gestión de Información en Salud de la carrera de Tecnología de la Salud. Educ Méd Super [serie en Internet]. 2008[citado 9 Ago 2008];22(1). Disponible en: http://bvs.sld.cu/revistas/ems/vol22_1_08/ems06108.htm

2. Ríos Massabot E, Fernández Viera RM, Jorge Pérez ER. Los registros médicos en Cuba. Rev Cubana Salud Pública [serie en Internet]. 2005[citado 9 Ago 2008; 31(4). Disponible en: http://scielo.sld.cu/scielo.php?script=sci arttext\&pid=S0864$\underline{34662005000400013 \& \operatorname{lng}=e s \& n r m=i s o}$

3. Vidal Ledo M, Fernández Oliva B, Alfonso Sánchez IR, Armenteros Vera I. Información, informática y estadísticas de salud: un perfil de la tecnología de la salud. ACIMED [serie en Internet]. 2004[citado10 Jul 2006]; 12(4). Disponible en: http://bvs.sld.cu/revistas/aci/vol12_4_04/aci08404.htm

4. Marín Milanés F, Torres Velazquez A. La información en la ciencia de la Información: tras las huellas de un concepto. ACIMED [serie en Internet]. 2005[ citado30 J ul 2006]; 13(5). Disponible en: http://bvs.sld.cu/revistas/aci/vol13_5_05/aci09505. htm

5. Rodríguez RJ, Gattinl C, Almeida G. El establecimiento de Sistemas de información en servicios de atención de salud. Guía para el análisis de requisitos, especificación de las aplicaciones y adquisición. Washington, D.C.: PAHO; 1999. 
6. Vidal Ledo M. Primera Estrategia para la Informatización del Sector de la Salud Pública Cubana [monografía en Internet]. 2007[citado7 Ago 2008]. Disponible en: http:// www.bvs.sld.cu/libros/prim_estrategia_inform/indice_p.htm

7. Vidal Ledo M. Modelo educativo para la gestión de información en salud [tesis]. La Habana: Escuela Nacional de Salud Pública; 2007.

8. Delgado Ramos A, Vidal Ledo M. Informática en la salud pública cubana. Rev Cubana Salud Pública [serie en Internet]. 2006 Sep[citado 9 Ago 2008]; 32(3). Disponible en: http://scielo.sld.cu/scielo.php?script=sci_arttext\&pid=S0864 34662006000300015\&lng=es\&nrm=iso

9. CESALC/UNESCO. Política para el cambio y el desarrollo de la Educación Superior [resumen ejecutivo]. París: UNESCO; 1995.

10. Horruitiner Silva P. La Universidad Cubana: el modelo de formación. La Habana: Editorial Félix Varela; 2006.

11. Vidal Ledo M. Diseño curricular del perfil de Información, Informática y Estadística de Salud de la carrera de Tecnología de la Salud [tesis]. La Habana: Centro de Enseñanza de Cibernética Aplicado a la Medicina (CECAM);2005.

12. Portal Pineda J. Estrategia educativa y de la motivación profesional para estudiantes del primer año de la carrera de Tecnología de la Salud [tesis]. La Habana: Escuela Nacional de Salud Pública; 2005.

13. Vogliotty A, Macchiarola V, Nicoletti S, Morales G. La compleja vinculación teoría-práctica en tendencias de formación docente [citado5 May 2006]. Disponible en: http://www. monografias.com/trabajos31/vinculacion-teoria-practica-formaciondocente/vinculacion -teoria-practica-formacion-docente.shtml

Recibido: 15 de septiembre de 2008.

Aprobado: 25 de septiembre de 2008.

María Vidal Ledo. Escuela Nacional de Salud Pública. Calle Línea Esq I. El Vedado 10400. La Habana, Cuba.

E-mail: mvidal@infomed.sld.cu 